\title{
Cathodic Voltage-controlled Electrical Stimulation Plus Prolonged Vancomycin Reduce Bacterial Burden of a Titanium Implant-associated Infection in a Rodent Model
}

\author{
Scott R. Nodzo MD, Menachem Tobias MS, Richard Ahn MD, \\ Lisa Hansen MS, Nicole R. Luke-Marshall PhD, Craig Howard BS, \\ Linda Wild MD, Anthony A. Campagnari PhD, Mark T. Ehrensberger PhD
}

Published online: 22 January 2016

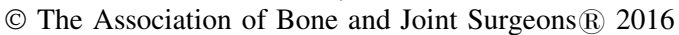

\begin{abstract}
Background Cathodic voltage-controlled electrical stimulation (CVCES) of titanium implants, either alone or combined with a short course of vancomycin, has previously been shown to reduce the bone and implant bacterial burden in a rodent model of methicillin-resistant Staphylococcus aureus (MRSA) implant-associated infection (IAI). Clinically, the goal is to achieve complete eradication of the IAI; therefore, the rationale for the present study was to evaluate the antimicrobial effects of combining CVCES with prolonged antibiotic therapy with the goal of decreasing the colony-forming units (CFUs) to undetectable levels.
\end{abstract}

\footnotetext{
The institution of one or more of the authors (MTE) has received, during the study period, funding from the Congressionally Directed Medical Research Program, Peer Reviewed Orthopedic Research Program, and the Bruce Holm Memorial Catalyst Fund. All ICMJE Conflict of Interest Forms for authors and Clinical Orthopaedics and Related Research ${ }^{\mathbb{R}}$ editors and board members are on file with the publication and can be viewed on request.

Clinical Orthopaedics and Related Research ${ }^{\mathbb{B}}$ neither advocates nor endorses the use of any treatment, drug, or device. Readers are encouraged to always seek additional information, including FDAapproval status, of any drug or device prior to clinical use. Each author certifies that his or her institution approved the animal protocol for this investigation and that all investigations were conducted in conformity with ethical principles of research.
}

S. R. Nodzo, M. Tobias, R. Ahn, C. Howard

Department of Orthopedics, State University of New York at Buffalo, Buffalo, NY, USA

L. Hansen, N. R. Luke-Marshall, A. A. Campagnari Department of Microbiology and Immunology, State University of New York at Buffalo, Buffalo, NY, USA

L. Wild

Department of Pathology and Anatomical Sciences, State

University of New York at Buffalo, Buffalo, NY, USA
Questions/purposes (1) In an animal MRSA IAI model, does combining CVCES with prolonged vancomycin therapy decrease bacteria burden on the implant and surrounding bone to undetectable levels? (2) When used with prolonged vancomycin therapy, are two CVCES treatments more effective than one? (3) What are the longer term histologic effects (inflammation and granulation tissue) of CVCES on the surrounding tissue?

Methods Twenty adult male Long-Evans rats with surgically placed shoulder titanium implants were infected with a clinical strain of MRSA (NRS70). One week after infection, the rats were randomly divided into four groups of five: (1) VANCO: only vancomycin treatment $(150 \mathrm{mg} /$ $\mathrm{kg}$, subcutaneous, twice daily for 5 weeks); (2) VANCO + 1STIM: vancomycin treatment (same as the VANCO group) coupled with one CVCES treatment $(-1.8 \mathrm{~V}$ for 1 hour on postoperative day [POD] 7); (3) VANCO + 2STIM: vancomycin treatment (same as the VANCO group) coupled with two CVCES treatments $(-1.8 \mathrm{~V}$ for 1 hour on POD 7 and POD 21); or (4) CONT: no treatment. On POD 42, the implant, bone, and peripheral blood were collected for CFU enumeration and histological analysis, where we compared $\mathrm{CFU} / \mathrm{mL}$ on the implants and bone among the groups. A pathologist, blinded to the experimental conditions, performed a semiquantitative analysis

M. T. Ehrensberger ( $\square)$

Department of Biomedical Engineering, State University of New

York at Buffalo, 162 Farber Hall, 3435 Main Street, Buffalo,

NY 14214, USA

e-mail: mte@buffalo.edu 
of inflammation and granulation tissue present in serial sections of the humeral head for animals in each experimental group.

Results The VANCO + 1STIM decreased the implant bacterial burden $($ median $=0$, range $=0-10 \mathrm{CFU} / \mathrm{mL}$ ) when compared with CONT $\left(\right.$ median $=5.7 \times 10^{4}$, range $=$ $4.0 \times 10^{3}-8.0 \times 10^{5} \mathrm{CFU} / \mathrm{mL}$; difference of medians $=$ $\left.-5.6 \times 10^{4} ; \mathrm{p}<0.001\right)$ and VANCO (median $=$ $4.9 \times 10^{3}$, range $=9.0 \times 10^{2}-2.1 \times 10^{4} \mathrm{CFU} / \mathrm{mL}$; difference of medians $\left.=-4.9 \times 10^{3} ; \mathrm{p}<0.001\right)$. The VANCO + 1STIM decreased the bone bacterial burden (median $=0$, range $=0-0 \mathrm{CFU} / \mathrm{mL})$ when compared with CONT $\left(\right.$ median $=1.3 \times 10^{2}$, range $=0-9.4 \times 10^{2} \mathrm{CFU} /$ $\mathrm{mL}$; difference of medians $\left.=-1.3 \times 10^{2} ; \mathrm{p}<0.001\right)$ but was not different from VANCO (median $=0$, range $=0$ $1.3 \times 10^{2} \mathrm{CFU} / \mathrm{mL}$; difference of medians $\left.=0 ; \mathrm{p}=0.210\right)$. The VANCO +2 STIM group had implant CFU (median $=$ 0 , range $\left.=0-8.0 \times 10^{1} \mathrm{CFU} / \mathrm{mL}\right)$ and bone $\mathrm{CFU}($ median $=$ 0 , range $\left.=0-2.0 \times 10^{1} \mathrm{CFU} / \mathrm{mL}\right)$ that were not different from the $\mathrm{VANCO}+1 \mathrm{STIM}$ treatment group implant CFU (median $=0$, range $=0-10 \mathrm{CFU} / \mathrm{mL}$; difference of medians $=$ $0 ; \mathrm{p}=0.334$ ) and bone $\mathrm{CFU}$ (median $=0$, range $=0-0$ $\mathrm{CFU} / \mathrm{mL}$; difference of medians $=0 ; \mathrm{p}=0.473$ ). The histological analysis showed no deleterious effects on the surrounding tissue as a result of the treatments.

Conclusions Using CVCES in combination with prolonged vancomycin resulted in decreased MRSA bacterial burden, and it may be beneficial in treating biofilm-related implant infections.

Clinical Relevance CVCES combined with clinically relevant lengths of vancomycin therapy may be a treatment option for IAI and allow for component retention in certain clinical scenarios. However, more animal research and human trials confirming the efficacy of this approach are needed before such a clinical recommendation could be made.

\section{Introduction}

Bacterial biofilm formation on retained orthopaedic components has been cited as a main cause for failure of irrigation and débridement without implant removal in the setting of chronic implant-associated infection (IAI) [22]. The complex glycocalyx structure created by the bacteria is adherent to the implant and highly resistant to antibiotic penetration, resulting in a limited success rate of irrigation and débridement without implant removal [5]. As a result of difficulties treating bacterial biofilms and the rise in drug-resistant bacteria, alternative therapeutic strategies must be investigated. Cathodic voltage-controlled electrical stimulation (CVCES) of titanium (Ti) implants has recently been shown to be a promising treatment against methicillin-resistant Staphylococcus aureus (MRSA) IAIs in both in vitro and in vivo studies [11, 19]. We recently reported results using a rodent model of MRSA IAI showing a $99.8 \%$ reduction in the implant bacterial burden when treated with CVCES of $-1.8 \mathrm{~V}$ versus $\mathrm{Ag} / \mathrm{AgCl}$ for 1 hour in combination with a 1-week course of antibiotics [19].

Although bacterial reduction was substantial using this novel methodology, no animals were completely infectionfree at the endpoints of these previous studies [11, 19]. Clinically, the goal is to achieve complete eradication of the IAI; therefore, the rationale for the present study was to evaluate the antimicrobial effects of combining CVCES with a prolonged antibiotic therapy with the goal of decreasing the colony-forming units (CFUs) to undetectable levels. In addition, although our previous studies showed CVCES had no immediate and no short-term deleterious histological effects on the surrounding tissues, it is unclear if CVCES has a deleterious effect on the surrounding bone that may manifest at later time points after stimulation.

We therefore asked: (1) In an animal MRSA IAI model, does combining CVCES with prolonged vancomycin therapy decrease bacteria burden on the implant and surrounding bone to undetectable levels? (2) When used with prolonged vancomycin therapy, are two CVCES treatments more effective than one? (3) What are the longer term histologic effects (inflammation and granulation tissue) of CVCES on the surrounding tissue?

\section{Materials and Methods}

All of the experimental protocols used in this study were reviewed and approved by the University at Buffalo Institutional Animal Care and Use Committee. Using a previously developed rodent MRSA IAI model [11, 19], this study sought to evaluate the antimicrobial and histological effects of treatment with vancomycin alone (VANCO), one treatment of CVCES combined with vancomycin (VANCO + 1STIM), and two treatments of CVCES combined with vancomycin (VANCO + 2STIM). In addition, a group that received no treatment served as controls (CONT). A power analysis, conducted in $\mathrm{R}$ statistical software (R Foundation for Statistical Computing, Vienna, Austria) using data from previous work with this stimulation animal model showing an implant CFU population SD of $1.2 \times 10^{6}$, determined five rodents should be used in each experimental group to detect a 99\% reduction in CFU at a power of $80 \%$ and $p$ value of 0.05 . 


\section{Bacteria}

All experiments were conducted with MRSA strain NRS70, a clinical respiratory isolate [16]. The minimal inhibitory concentration for vancomycin against NRS70 was determined by the clinical microbiology laboratory at the Buffalo VA Hospital to be $0.5 \mu \mathrm{g} / \mathrm{mL}$. Bacteria were suspended in tryptic soy broth supplemented with $0.25 \%$ glucose to an optical density at $600 \mathrm{~nm}\left(\mathrm{OD}_{600}\right)$ of 0.1 (corresponding to approximately $10^{7} \mathrm{CFU}$ per $\mathrm{mL}$ ) and diluted $1: 10$ in physiologic saline $(0.9 \% \mathrm{NaCl}, \mathrm{pH} 7.2)$ to generate the inoculum.

\section{Surgical Implantation and Infection}

A total of 20 adult Long-Evans rats (males, 175-200 g/8-9 weeks) were acquired from Harlan Laboratories (Indianapolis, IN, USA). A standard procedure previously described was used to surgically place the implant $[11,19]$. In brief, after successful induction of anesthesia using ketamine, xylazine, and acepromazine, the ventral periaxillary area was shaved, sterilized with chlorhexidine and alcohol, and injected with local anesthetic (bupivacaine and lidocaine mix) and analgesic (buprenorphine) subcutaneously. An oblique 1-cm skin incision was made over the shoulder, and sharp dissection through the subcutaneous fat and superficial muscle layers was carried down to expose the anterior portion of the rotator cuff. The anterior one-third of the rotator cuff was sharply incised with the shoulder in maximal abduction and external rotation to expose the humeral head. The humeral head was dislocated, and a 1.7-mm drill bit was used to drill through the center of the humeral head and out through the lateral cortex. The canal created was inoculated with approximately $10^{5} \mathrm{CFU}(0.1 \mathrm{cc})$ of NRS70. After inoculation, a custom $1.6 \mathrm{~mm} \times 16-\mathrm{mm}$ sterilized $\mathrm{Ti}$ rod (Titanium Industries, Inc, Rockaway, NJ, USA) was implanted in the canal so that it sat flush with the articular surface of the humeral head and extended slightly beyond the lateral cortex of the humerus (Fig. 1). After implantation, the deep tissue and skin incisions were closed using 4-0 Webcryl (Patterson Veterinary, Devens, MA, USA), and carprofen was administered for postoperative pain management. Rats were maintained 6 days postoperatively for establishment of an IAI.

\section{Treatment Protocol}

A total of 20 animals were randomly assigned to four experimental groups: VANCO, VANCO + 1STIM, VANCO + 2STIM, or CONT. Each experimental group

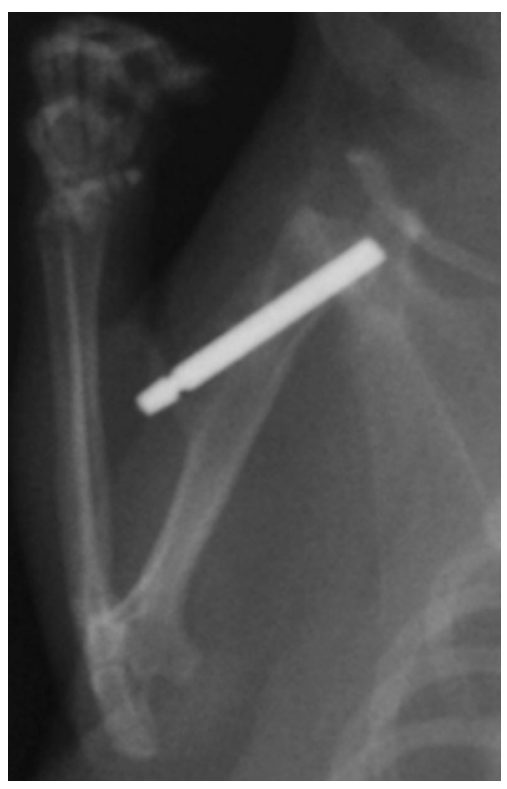

Fig. 1 This radiograph shows the placement of the Ti implant in the rat humerus. (Reprinted from Nodzo S, Tobias M, Hansen L, LukeMarshall NR, Cole R, Wild L, Campagnari AA, Ehrensberger MT. Cathodic electrical stimulation combined with vancomycin enhances treatment of methicillin-resistant Staphylococcus aureus implantassociated infections. Clin Orthop Relat Res. 2015;473:2856-2864, with kind permission from Springer Science and Business Media.)

consisted of five animals. On postoperative Day (POD) 6, the rats in the VANCO, VANCO + 1STIM, and VANCO + 2STIM groups started receiving subcutaneous injections of vancomycin at $150 \mathrm{mg} / \mathrm{kg}$ (V8138; Sigma-Aldrich, St Louis, MO, USA) twice a day. This dosing was derived from other experimental models [1, 4, 15, 21, 25] and previous work in our laboratory [19]. On POD 7, all animals were anesthetized and the skin over the lateral cortex of the humerus was incised to expose the end of the Ti rod. Subsequently, an $\mathrm{Ag} / \mathrm{AgCl}$ pellet reference electrode (E242ML; In Vivo Metric, Healdsburg, CA, USA) and a dual platinum wire counterelectrode (CHI115; CH Instruments, Austin, TX, USA) were placed subcutaneously at an incision site immediately adjacent to the incision site providing access to the $\mathrm{Ti}$ rod without disruption or manipulation of the intraarticular IAI (Fig. 2). In the $\mathrm{VANCO}+1 \mathrm{STIM}$ and VANCO $+2 \mathrm{STIM}$ groups, the electrodes were connected to a potentiostat (Ref 600; Gamry Instruments, Warminster, PA, USA) that provided CVCES of $-1.8 \mathrm{~V}$ (all voltages are versus $\mathrm{Ag} / \mathrm{AgCl}$ unless otherwise noted) to the $\mathrm{Ti}$ rod (working electrode) for 1 hour. Animals in the VANCO and CONT groups had the electrodes placed, but no stimulation was delivered during the sham procedure. After the stimulation/sham procedure, all electrodes were removed, incisions sutured, and the animals were returned to their cages. Animals in the VANCO, VANCO + 1STIM, and VANCO + 2STIM 


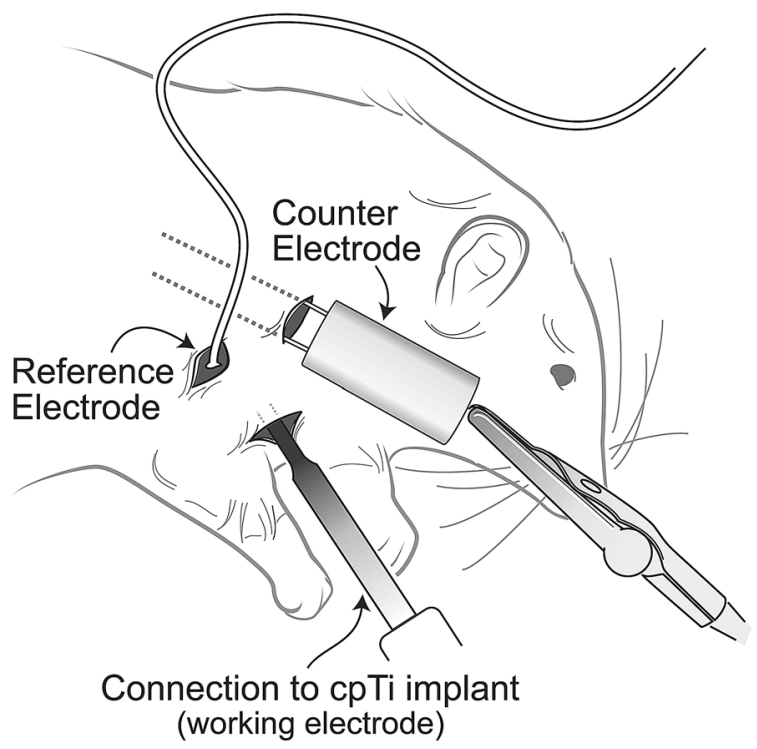

Fig. 2 This schematic shows the in vivo stimulation using a threeelectrode system. The Ti implant (working electrode) was accessed through internal cortex incision, whereas $\mathrm{Ag} / \mathrm{AgCl}$ (reference) and platinum (counter) electrodes were placed subcutaneously adjacent to the Ti implant. (Reprinted from Ehrensberger MT, Tobias ME, Nodzo SR, Hansen LA, Luke-Marshall NR, Cole RF, Wild LM, Campagnari AA. Cathodic voltage-controlled electrical stimulation of titanium implants as treatment for methicillin-resistant Staphylococcus aureus periprosthetic infection. Biomaterials. 2015;41:97-105, with permission from Elsevier.)

groups continued to receive the $150-\mathrm{mg} / \mathrm{kg}$ twice-daily subcutaneous injections of vancomycin. On POD 21 the VANCO + 2STIM group underwent an additional 1-hour CVCES using the same protocol as previously described. After this additional procedure, the rodents continued to receive the $150-\mathrm{mg} / \mathrm{kg}$ twice-daily subcutaneous injections of vancomycin.

On POD 42, all animals were anesthetized using isoflurane and euthanized by cardiac puncture for harvesting of specimens. The Ti implant was extracted for enumeration of CFU. The humeral head and diaphysis were harvested for enumeration of CFU and histological analysis. Additionally, peripheral venous blood samples were collected by venipuncture just before cardiac puncture, which confirmed that none of the animals were septic at the time of euthanasia.

\section{Enumeration of $\mathrm{CFU}$}

The Ti rod and the proximal segment of the humeral head collected at the end of the studies were individually rinsed with sterile phosphate-buffered saline (PBS), immersed in PBS- $0.1 \%$ saponin, and subjected to a sonicating water bath for 10 minutes. Viable CFUs were enumerated from the Ti, humeral head bone specimens, and peripheral blood samples by duplicate plating separate $100-\mu \mathrm{L}$ aliquots of the original $1 \mathrm{~mL}$ sonicated volume in serial 10-fold dilutions onto Mueller Hinton agar plates and incubating at $37^{\circ} \mathrm{C}$ in $5 \% \mathrm{CO}_{2}$ for 18 hours.

\section{Histological Evaluation}

The humeral head and diaphysis bone specimens were immediately fixed in $10 \%$ neutral buffered formalin phosphate (Fisher Scientific, Waltham, MA, USA) after harvest. Briefly, tissue samples consisting of cross-sections through the rat humerus were obtained perpendicular to the axis of the implant canal from proximal, mid-, and distal locations, decalcified for approximately 24 hours, paraffin wax-embedded, cut to $5-\mu \mathrm{m}$ thick serial sections, and stained using hematoxylin and eosin. The serial sections from the animals in all experimental groups were evaluated by a pathologist (LW) under blinded review for any light microscopic evidence of cell damage or necrosis in the bone and surrounding tissues. The sections were also evaluated using a semiquantitative scale and each section was assigned a score (1-4) based on the degree of inflammatory response noted in the implant canal wall $(1=$ no inflammation noted, 2 = slight inflammation, $3=$ moderate inflammation, $4=$ extensive inflammation). Measurements of granulation tissue thickness around the implant canal were also tabulated.

\section{Statistical Analysis}

A Kruskal-Wallis nonparametric test followed by serial Mann-Whitney post hoc tests with a Bonferroni correction were used to compare the log-transformed CFU data across all experimental groups. As a result of the Bonferroni correction, a $\mathrm{p}$ value $<0.0125$ was considered statistically significant for the Mann-Whitney tests. All statistical tests were performed with Graphpad Prism 6 software (La Jolla, CA, USA).

\section{Results}

The VANCO + 1STIM treatment decreased the implant bacterial burden $($ median $=0$, range $=0-10 \mathrm{CFU} / \mathrm{mL}$ ) when compared with CONT $\left(\right.$ median $=5.7 \times 10^{4}$, range $=$ $4.0 \times 10^{3}-8.0 \times 10^{5} \mathrm{CFU} / \mathrm{mL}$; difference of medians $=$ $-5.6 \times 10^{4} ; \mathrm{p}<0.001$ ) and VANCO (median $=$ $4.9 \times 10^{3}$, range $=9.0 \times 10^{2}-2.1 \times 10^{4} \mathrm{CFU} / \mathrm{mL}$; difference of medians $\left.=-4.9 \times 10^{3} ; \mathrm{p}<0.001\right)($ Fig. 3A). The VANCO + 1STIM treatment also decreased the bone 


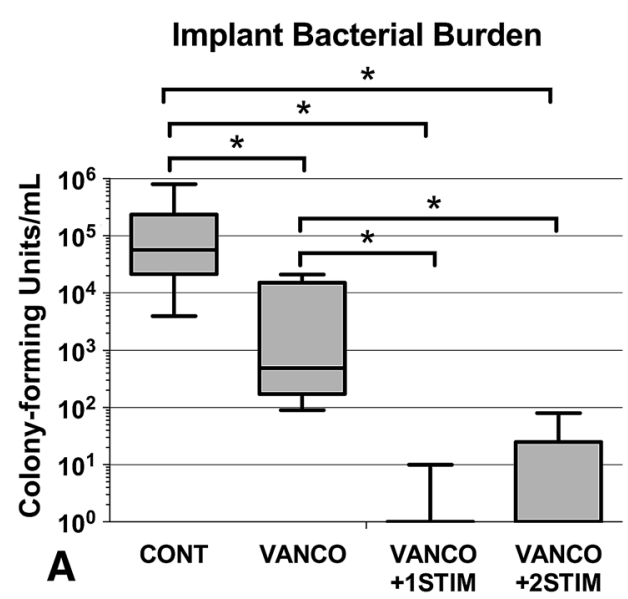

Fig. 3A-B These plots show the CFU enumerated from the Ti implant (A) and bone (B) for the control (CONT), vancomycin alone (VANCO), one cathodic voltage-controlled electrical stimulation and vancomycin (VANCO + 1STIM), and two cathodic voltage-controlled electrical stimulations and vancomycin (VANCO + 2STIM)

bacterial burden $($ median $=0$, range $=0-0 \mathrm{CFU} / \mathrm{mL}$ ) when compared with CONT $\left(\right.$ median $=1.3 \times 10^{2}$, range $=0$ $9.4 \times 10^{2} \mathrm{CFU} / \mathrm{mL}$; difference of medians $=-1.3 \times 10^{2}$; $\mathrm{p}<0.001$ ) but was not different from the VANCO (median $=$ 0 , range $=0-1.3 \times 10^{2} \mathrm{CFU} / \mathrm{mL} ;$ difference of medians $=$ $0 ; p=0.210$ ) (Fig. 3B). Importantly, within the VANCO + 1STIM group, four of the five animals had undetectable implant CFU and all animals in this group had undetectable bone CFU.

The VANCO +2 STIM treatment group had implant $\mathrm{CFU}$ (median $=0$, range $=0-8.0 \times 10^{1} \mathrm{CFU} / \mathrm{mL}$ ) and bone $\mathrm{CFU}$ (median $=0$, range $=0-2.0 \times 10^{1} \mathrm{CFU} / \mathrm{mL}$ ) that were not different from the VANCO + 1STIM treatment group implant $\mathrm{CFU}$ (median $=0$, range $=0-10 \mathrm{CFU} / \mathrm{mL}$; difference of medians $=0 ; p=0.334$ ) (Fig. 3A) and bone $\mathrm{CFU}$ (median $=0$, range $=0-0 \mathrm{CFU} / \mathrm{mL}$; difference of medians $=0 ; \mathrm{p}=0.473$ ) (Fig. 3B).

Review of histopathologic sections revealed no deleterious histological effects of treatment. Microscopic sections of decalcified bone from specimens in all experimental groups of rats showed defects in the bone tissue from the implants (Fig. 4A) that extend from the subchondral region beneath the articular surface to the proximal diaphysis. Variable amounts of granulation tissue (GT) and acute and chronic inflammation were present surrounding the implant defects (ID) and extended focally into the surrounding bone tissue (BT). The CONT group (Fig. 4B) showed thick walls of granulation tissue surrounding the implant defect with an average thickness of $623 \mu \mathrm{m}$. This granulation tissue contained abundant purulent inflammatory exudate with areas of abscess formation and had an average inflammatory score of $3.4 \pm 0.9$, indicating a moderate to extensive

\section{Bone Bacterial Burden}

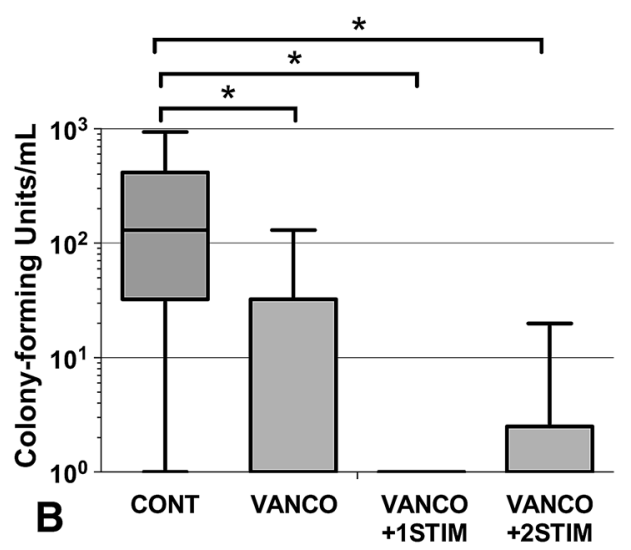

groups. The data sets are presented as box-and-whisker plots where the whiskers represent the CFU range, box edges represent the upper and lower quartiles, and the band inside the box is the median CFU value. The CFUs are presented on a log scale. *Statistically significant differences where $\mathrm{p}<0.0125$.

inflammatory response. The VANCO group (Fig. 4C) had less extensive amounts of surrounding granulation tissue with an average thickness of $244 \mu \mathrm{m}$ and received an average inflammatory score of $2.7 \pm 0.4$, indicating a moderate inflammatory response. The VANCO + 1STIM and VANCO + 2STIM groups showed a similar and milder inflammatory response (representative section shown in Fig. 4D) with average scores of $2.2 \pm 0.2$ and $2.1 \pm 1.0$, respectively, and average granulation tissue thicknesses of $187 \mu \mathrm{m}$ and $176 \mu \mathrm{m}$, respectively. There was no evidence of bone necrosis in any of the experimental groups.

\section{Discussion}

Our previous work has shown that a single 1-hour application of $-1.8 \mathrm{~V}$ CVCES to Ti implants reduces the MRSA CFU recovered from the bone and implant by one to two orders of magnitude when assessed immediately after the stimulation [11]. Followup work showed that a single stimulation alone does not have sustained antimicrobial effects when assessed 1 week poststimulation [19]. However, combining the single CVCES with a 1-week time course of vancomycin produced a $99.8 \%$ reduction, but still a detectable level, of bone and implant CFU [19]. The present study was conducted to evaluate if a prolonged 5-week course of vancomycin therapy combined with either one or two CVCES treatments is able to further reduce the bacterial burden in an IAI model to undetectable levels. In addition, this study evaluated the histological effects of CVCES on the surrounding bone at a longer followup to determine if late deleterious bony manifestations were produced. 


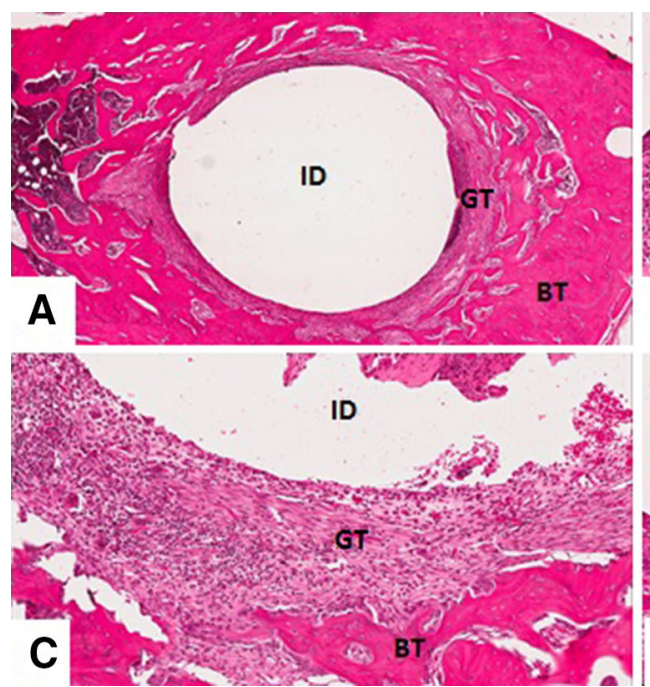

Fig. 4A-D These images show representative histological sections of the rat humerus after Ti implant removal. (A) Representative lowmagnification view illustrating the orientation of the ID, surrounding GT, and the peripheral BT (Stain, hematoxylin and eosin; original magnification, $\times 5$ ). Higher magnification views illustrate the variation in the granulation tissue for the no treatment control group (B; Stain: hematoxylin and eosin; original magnification, $\times 20$ ), the

This work does have its limitations. First, these results are from an in vivo rodent model of IAI, and the results and techniques reported may not completely translate to the clinical setting. Second, we did not confirm biofilm formation on the prosthesis before the electrical stimulation protocol and it is recognized that clinically IAI might appear at a time later than POD 6 when more robust biofilms might be present. However, the results suggest antibiotic therapy alone did not effectively treat the implant bacterial burden in this model, which is consistent with biofilm-associated orthopaedic infections seen clinically [26]. We also must point out that the undetectable CFU levels presented in this study may not necessarily mean the infection was eradicated. It is possible that our sonication method for biofilm removal, although done in the presence of saponin, may have left adherent bacteria on the implant and bone and future studies should also include a method for ruling out this possibility. In this study we also only report outcomes at a 5-week time point and it is possible the IAI reached undetectable CFU levels before this selected endpoint, which could indicate that the length of antibiotic therapy may be able to be shortened when it is combined with CVCES. In addition, the semiquantitative histological scoring system implemented has not undergone rigorous validation; however, a single pathologist who was blinded to the experimental conditions performed the histological review. Finally, this work evaluated CVCES for commercially pure Ti implants, whereas titanium- $6 \%$ aluminum- $4 \%$ vanadium alloy is more

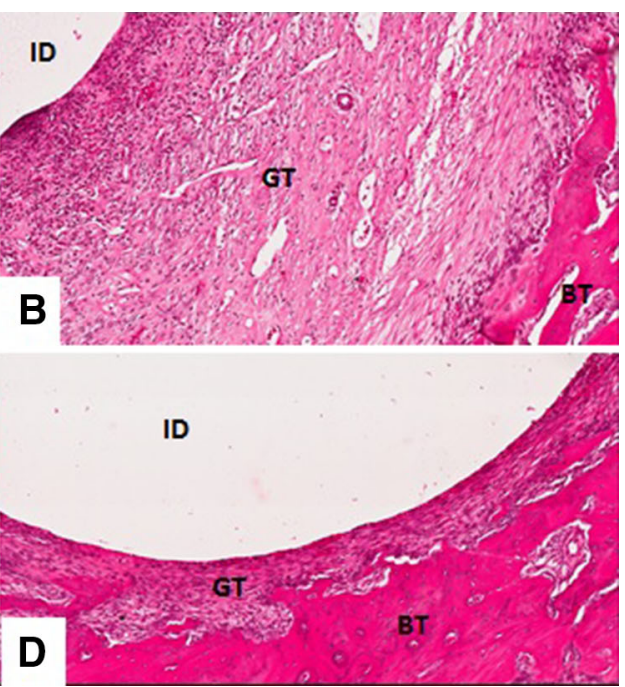

vancomycin alone $(\mathbf{C}$; Stain, hematoxylin and eosin; original magnification, $\times 20$ ), and combined stimulation and vancomycin treatment (D; Stain: hematoxylin and eosin; original magnification, $\times 20$ ). The combined one and two cathodic voltage-controlled electrical stimulation treatment groups displayed similar tissue responses and D characterizes this response.

commonly used as an orthopaedic implant material. However, the passive surface film on both of these materials is largely dictated by titanium-oxide and previous studies have shown these metals have very similar surface oxide behavior and voltage-dependent electrochemical properties [2]. It is also important to emphasize that most other metals used for orthopaedic implants such as stainless steel and cobalt-chromium-molybdenum are passivated with surface oxide films that also display voltage-dependent electrochemical properties $[12,14,20]$. Therefore, although the results presented here are for commercially pure $\mathrm{Ti}$, the antimicrobial strategy of using CVCES to control the voltage-dependent interfacial electrochemical properties of other metal orthopaedic implants may be broadly applicable and should be evaluated in future studies.

A single, 1-hour CVCES at $-1.8 \mathrm{~V}$ in combination with a 5-week course of vancomycin therapy effectively decreased the implant and bone bacterial burden and in $80 \%$ of the animals reduced the CFU to undetectable levels. The reduction in bone tissue bacterial burden was similar to that of just vancomycin treatment, but synergistic reductions of the implant bacterial burden were noted for the combined treatment. These results are encouraging and expand on previous work that showed a synergistic effect of vancomycin therapy and CVCES [11, 19]. These reported effects of vancomycin alone are consistent with clinical observations in which the tissue infection is often well treated by antibiotics, whereas 
implant-associated infections usually fail treatment without implant removal. The present results suggest that CVCES directly altered the viability of the implant bacteria and/or rendered them more susceptible to the antibiotics. This could be the result of disruption of the biofilm to release bacteria in the more vulnerable planktonic form or through a molecular or microenvironmental mechanism that is currently unknown. Although there have been numerous in vitro reports on the antimicrobial effects of direct electrical simulation with or without combination with antibiotics $[3,6,8,9,17,18,24]$, there are only a few in vivo reports $[7,13,23]$. In contrast to the other in vivo models using electrical stimulation to treat implant-associated infections [7, 13, 23], CVCES uses a three-electrode configuration that allows us to precisely control the cathodic potential of the titanium implant [10, 11]. Although the exact antimicrobial mechanism of CVCES is not well understood, it is believed that cathodic modulation of Ti's voltage-dependent properties is one of the main driving factors for the robust antibacterial effects we have observed in our experiments [10,11, 19].

The application of a second CVCES 2 weeks after the first CVCES did not show an additional antimicrobial benefit when evaluated at the endpoint of this study. This is likely related to the fact that very low levels of CFU were detected with just the combine single CVCES. However, these results do not necessarily preclude further exploration of multiple CVCES treatments. For example, it is possible that multiple treatments with CVCES could allow for greater reductions in the bacterial burden, or perhaps complete clearance, of the IAI at an earlier time point. The current study was not designed to assess this possibility, but future studies should focus on this aspect.

The histological outcomes revealed no signs of bone necrosis. These results are consistent with our previous work evaluating the bone histologic response to CVCES at earlier time points [11, 19], adding further evidence to the benign nature of this treatment on the adjacent bone. The thickest inflammatory granulation tissues observed for the CONT group are consistent with an ongoing and persistent infection, whereas the groups receiving treatment for the infection had diminished granulation tissue thickness and cellular inflammatory response. In particular, the thinner granulation tissue and lower inflammation score noted for the CVCES groups, which included some animals with undetectable CFU levels, may suggest a resolving host inflammatory response that could possibly be associated with the clearance of the infection. Other in vivo studies that have evaluated constant current electrical stimulation of intramedullary stainless steel implants for treatment of Staphylococcus epidermidis osteomyelitis in rabbits have reported bone discoloration after 21 days of treatment [7]. However, there was no histological assessment of the tissues in this previous study. Importantly, in the present and past CVCES in vivo studies, there have been no reports of bone discoloration or histologically discernible deleterious effects on the bone tissue when evaluated at 5 weeks, 1 week, and immediately after the CVCES $[11,19]$.

Using CVCES in combination with prolonged vancomycin resulted in decreased MRSA bacterial burden in an IAI rodent model, and importantly, CVCES treatments were not associated with any deleterious histological changes in the surrounding bone tissue. These outcomes suggest that CVCES could be a potential treatment option for IAI and allow for component retention in certain clinical scenarios. However, more animal research and human trials confirming the efficacy of this approach are needed before such a clinical recommendation could be made. Future work focused on the advanced biomechanical analysis of stimulated implants, the effects of electrical stimulation on commonly used orthopaedic alloys, and the optimal timing, magnitude, and duration of the stimulation will be necessary before this technology moves into the clinical setting.

\section{References}

1. Aronoff GR, Sloan RS, Dinwiddie CB Jr, Glant MD, Fineberg NS, Luft FC. Effects of vancomycin on renal function in rats. Antimicrob Agents Chemother. 1981;19:306-308.

2. Bearinger JP, Orme CA, Gilbert JL. In situ imaging and impedance measurements of titanium surfaces using AFM and SPIS. Biomaterials. 2003;24:1837-1852.

3. Blenkinsopp SA, Khoury AE, Costerton JW. Electrical enhancement of biocide efficacy against Pseudomonas aeruginosa biofilms. Appl Environ Microbiol. 1992;58:3770-3773.

4. Chilukuri DM, Shah JC. Local delivery of vancomycin for the prophylaxis of prosthetic device-related infections. Pharm Res. 2005;22:563-572.

5. Costerton JW, Cheng KJ, Geesey GG, Ladd TI, Nickel JC, Dasgupta M, Marrie TJ. Bacterial biofilms in nature and disease. Annu Rev Microbiol. 1987;41:435-464.

6. Costerton JW, Ellis B, Lam K, Johnson F, Khoury AE. Mechanism of electrical enhancement of efficacy of antibiotics in killing biofilm bacteria. Antimicrob Agents Chemother. 1994;38:2803-2809.

7. Del Pozo JL, Rouse MS, Euba G, Kang CI, Mandrekar JN, Steckelberg JM, Patel R. The electricidal effect is active in an experimental model of Staphylococcus epidermidis chronic foreign body osteomyelitis. Antimicrob Agents Chemother. 2009;53:4064-4068.

8. Del Pozo JL, Rouse MS, Mandrekar JN, Sampedro MF, Steckelberg JM, Patel R. Effect of electrical current on the activities of antimicrobial agents against Pseudomonas aeruginosa, Staphylococcus aureus, and Staphylococcus epidermidis biofilms. Antimicrob Agents Chemother. 2009;53:35-40.

9. Del Pozo JL, Rouse MS, Mandrekar JN, Steckelberg JM, Patel R. The electricidal effect: reduction of Staphylococcus and Pseudomonas biofilms by prolonged exposure to low-intensity electrical current. Antimicrob Agents Chemother. 2009;53:41-45.

10. Ehrensberger MT, Gilbert JL. The effect of static applied potential on the 24 hour impedance of commercially pure titanium in simulated biological conditions. J Biomed Mater Res B Appl Biomater. 2010;93:106-112. 
11. Ehrensberger MT, Tobias ME, Nodzo SR, Hansen LA, LukeMarshall NR, Cole RF, Wild LM, Campagnari AA. Cathodic voltage-controlled electrical stimulation of titanium implants as treatment for methicillin-resistant Staphylococcus aureus periprosthetic infections. Biomaterials. 2015;41:97-105.

12. Gettens RT, Gilbert JL. The electrochemical impedance of polarized 316L stainless steel: structurepopertyasorption correlation. J Biomed Mater Res A. 2009;90:121-132.

13. Gilotra M, Griffith C, Schiavone J, Nimmagadda N, Noveau J, Ludwig SC. Capacitive coupling reduces instrumentation-related infection in rabbit spines: a pilot study. Clin Orthop Relat Res. 2012;470:1646-1651.

14. Haeri M, Goldberg S, Gilbert JL. The voltage-dependent electrochemical impedance spectroscopy of CoCrMo medical alloy using time-domain techniques: generalized Cauchy-Lorentz, and KWW-Randles functions describing non-ideal interfacial behaviour. Corros Sci. 2011;53:582-588.

15. Karau MJ, Schmidt-Malan SM, Greenwood-Quaintance KE, Mandrekar JN, Cai J, Pierce WM, Merten K, Patel R. Treatment of methicillin-resistant Staphylococcus aureus experimental osteomyelitis with bone-targeted vancomycin. SpringerPlusv. 2013;2:1-8.

16. Kuroda M, Ohta T, Uchiyama I, Baba T, Yuzawa H, Kobayashi I, Cui L, Oguchi A, Aoki K-I, Nagai Y, Lian J, Ito T, Kanamori M, Matsumaru H, Maruyama A, Murakami H, Hosoyama A, Mizutani-Ui Y, Takahashi NK, Sawano T, Inoue R-I, Kaito C, Sekimizu K, Hirakawa H, Kuhara S, Goto S, Yabuzaki J, Kanehisa M, Yamashita A, Oshima K, Furuya K, Yoshino C, Shiba T, Hattori M, Ogasawara N, Hayashi H, Hiramatsu K. Whole genome sequencing of meticillin-resistant Staphylococcus aureus. Lancet. 2001;357:1225-1240.

17. Mohn D, Zehnder M, Stark WJ, Imfeld T. Electrochemical disinfection of dental implants-a proof of concept. PLoS One. 2011;6:e16157.

18. Niepa THR, Gilbert JL, Ren D. Controlling Pseudomonas aeruginosa persister cells by weak electrochemical currents and synergistic effects with tobramycin. Biomaterials. 2012;33:73567365 .

19. Nodzo S, Tobias M, Hansen L, Luke-Marshall NR, Cole R, Wild L, Campagnari AA, Ehrensberger MT. Cathodic electrical stimulation combined with vancomycin enhances treatment of methicillin-resistant Staphylococcus aureus implant-associated infections. Clin Orthop Relat Res. 2015;473:2856-2864.

20. Pound BG. Passive films on metallic biomaterials under simulated physiological conditions. $J$ Biomed Mater Res A. 2014;102:1595-1604.

21. Rouse MS, Piper KE, Jacobson M, Jacofsky DJ, Steckelberg JM, Patel R. Daptomycin treatment of Staphylococcus aureus experimental chronic osteomyelitis. $J$ Antimicrob Chemother. 2006;57:301-305.

22. Scott IR, Stockley I, Getty CJ. Exchange arthroplasty for infected knee replacements. A new two-stage method. J Bone Joint Surg Br. 1993;75:28-31.

23. Van Der Borden AJ, Maathuis PG, Engels E, Rakhorst G, Van Der Mei HC, Busscher HJ, Sharma PK. Prevention of pin tract infection in external stainless steel fixator frames using electric current in a goat model. Biomaterials. 2007;28:2122-2126.

24. Van Der Borden AJ, Van Der Mei HC, Busscher HJ. Electriccurrent-induced detachment of Staphylococcus epidermidis from surgical stainless steel. J Biomed Mater Res B Appl Biomater. 2004;68:160-164.

25. Vaudaux P, Francois P, Bisognano C, Li D, Lew DP, Schrenzel J. Comparative efficacy of daptomycin and vancomycin in the therapy of experimental foreign body infection due to Staphylococcus aureus. J Antimicrob Chemother. 2003;52:89-95.

26. Zeller V, Durand F, Kitzis MD, Lhotellier L, Ziza JM, Mamoudy $\mathrm{P}$, Desplaces N. Continuous cefazolin infusion to treat bone and joint infections: clinical efficacy, feasibility, safety, and serum and bone concentrations. Antimicrob Agents Chemother. 2009;53:883-887. 